16. Rouch L, Cestac P, Hanon O et al (2015) Antihypertensive drugs, prevention of cognitive decline and dementia: a systematic review of observational studies, randomized controlled trials and metaanalyses, with discussion of potential mechanisms. CNSDrugs 29:113-130

17. Schröder J, Pantel J (2011) Die leichte kognitive Beeinträchtigung. Klinik, Diagnostik, Therapie und Prävention im Vorfeld der Alzheimer-Demenz. Schattauer, Stuttgart

18. Schröder J, Pantel J (2016) Neuroimaging of hippocampal atrophy in early recognition of Alzheimer's disease - a critical appraisal after two decades of research. Psychiatry Res 247:71-78

19. Singler K, Gurlit S et al (2014) Delir (akute Verwirrtheit). In: Pantel J, Schröder J, Bollheimer C (Hrsg) Praxishandbuch Altersmedizin. Geriatrie Gerontopsychiatrie - Gerontologie. Kohlhammer, Stuttgart, S275-298

20. Stranks EK, Crowe SF (2014) The acute cognitive effects of zopiclone, zolpidem, zalepon, and eszopiclone: a systematic review and metaanalysis. JClin Exp Neuropsychol 36:691-700

21. Wehling M (2016) How to use the FORTA ("Fit fOR The Aged") list to improve pharmacotherapy in the elderly. Drug Res 66:57-62

22. Wehling M, Burkhardt H (2013) Arzneitherapie für Ältere, 3. Aufl. Springer, Heidelberg

\title{
Erratum
}

Internist 2016 $57: 1036$

DOI 10.1007/s00108-016-0124-9

Online publiziert:22. August 2016

(c) Springer-Verlag Berlin Heidelberg 2016

(1) CrossMark

\section{U. R. Fölsch ${ }^{1}$ G. Hasenfuß ${ }^{2}$ - H.-F. Spies ${ }^{3}$ W. Wesiack • F. Faulbaum ${ }^{5}$}

'Klinik für Innere Medizin 1, Universitätsklinikum Schleswig-Holstein (UKSH), Campus Kiel, Kiel, Deutschland

${ }^{2}$ Klinik für Kardiologie und Pneumologie, Universitätsmedizin Göttingen, Göttingen, Deutschland

${ }^{3}$ Berufsverband Deutscher Internisten (BDI), Wiesbaden, Deutschland

${ }^{4}$ Hamburg, Deutschland

${ }^{5}$ Sozialwissenschaftliches Umfragezentrum GmbH, Duisburg, Deutschland

\section{Erratum zu: Flucht und Migration. Eine Herausforderung für die Medizin in Deutschland}

Erratum zu:

Internist (2016) 57: 822

DOI: 10.1007/s00108-016-0103-1

Bei der Angabe, wie viele Mitglieder sich an der Umfrage beteiligten, ist ein Fehler aufgetreten. Die richtige Angabe lautet wie folgt: Insgesamt konnten sich 32.062 Mitglieder an der Befragung beteiligen. Die Redaktion bittet um Beachtung der korrekten Anzahl.

\section{Korrespondenzadresse}

\section{Prof. Dr. U. R. Fölsch}

Klinik für Innere Medizin 1, Universitätsklinikum Schleswig-Holstein (UKSH), Campus Kiel Schittenhelmstr. 12, 24105 Kiel, Deutschland urfoelsch@1med.uni-kiel.de
Die Online-Version des Originalbeitrags finden Sie unter http://dx.doi.org/10.1007/s00108016-0103-1. 